\title{
BRIEF - SYNOPSIS OF THE HUMAN IMMUNODEFICIENCY VIRUS (HIV) SCREENING AND TESTING GUIDE
}

\author{
GALE-ROWE M, DODDS J, PAQUETTE D, WONG T ON BEHALF OF THE PHAC EXPERT WORKING \\ GROUP*
}

KEY WORDS: HIV/AIDS, SCREENING, HIV TESTING

\section{Structured Abstract}

\section{BACKGROUND}

The Public Health Agency of Canada (PHAC) estimates that, in 2011, 25\% of people living with HIV in Canada were undiagnosed. Hesitation to seek testing may arise from fear, stigma and discrimination associated with the HIV diagnosis and related risk behaviours. This guide is designed to complement existing efforts to support care providers involved in HIV testing, in order to reduce the number of undiagnosed HIV infections in Canada.

\section{APPROACH}

PHAC commissioned a literature review and consulted with provinces and territories, and key stakeholders, including people living with HIVIAIDS, academics, nurses, physicians, professional associations, nongovernmental organizations, policy-makers, community workers, and legal and ethical experts. As a result, the recommendations outlined in the guide are based on the most up-to-date evidence and expert opinion.

\section{SCREENING AND TESTING GUIDE}

The consideration and discussion of HIV testing should be made a component of routine periodic medical care. Offering HIV testing routinely can help normalize testing, and address the multiple barriers to reducing the number of undiagnosed cases in Canada. Begin with a brief explanation to the client on how HIV is transmitted: through unprotected sex, the sharing of drug-use equipment, and from a pregnant mother to her child. Clients can then consider their own situation and indicate whether they would like to have an HIV test. Upon request, a risk assessment may be conducted. As with other tests, testing is voluntary and verbal consent is sufficient.

Negative test results provide an opportunity to remind clients of those practices that can help them maintain an HIV-negative status. There are a range of referrals and resources available to assist clients in reducing at-risk activities and maintaining a negative status. Those who are part of a couple should be encouraged to discuss HIV testing with their partners so they're not unknowingly involved in a serodiscordant relationship.

Positive test results should always be provided in person and ideally by the initial care provider who has information resources and support referrals at the ready. An HIV positive diagnosis can be difficult news; it is important to take the time to discuss the results and answer any questions the client might have. Focus on positive messages by highlighting advances in HIV care, treatment and support. Note that HIV is now considered a chronic illness, and people living with HIV can live long, active and healthy lives. Advise the client about strategies for managing HIV and link them to care. Provide risk reduction information to prevent transmission. Make the client aware that positive test results will be shared confidentially with the local public health 
department, which can assist in notifying previous and current partners of the need to be tested while protecting the client's anonymity and privacy. Strategies for informing past, current and future partners can be reviewed. If not already completed with the HIV test, clients should be tested for other STIs, hepatitis B and C, and tuberculosis.

\section{Introduction}

There is a critical need to optimize opportunities for care providers to offer HIV testing to people living in Canada; undiagnosed cases are missed opportunities to reduce HIV transmission and improve productivity, disease prognosis and quality of life for people living with HIV. The Public Health Agency of Canada estimates that, in 2011, $25 \%$ of people living with HIV in Canada were unaware of their infection (1).

There are several benefits to reducing the number of undiagnosed HIV infections. A negative test result is an opportunity for clients to take an active role in remaining HIV negative. Individuals who receive their diagnosis earlier and initiate Highly Active Antiretroviral Therapy (HAART) can have reduced morbidity and mortality associated with HIV infection and disease progression compared with those who do not receive a timely diagnosis (2) Advances in HIV treatment have slowed the progression of the disease to such a degree that HIV infection is now understood to be a chronic, manageable condition enabling more people with HIV to live healthy, long, and active lives.

Those who test positive are more likely to take measures that prevent the onward spread of HIV (3). Emerging evidence is demonstrating that reduced individual viral load as a result of early initiation of HAART, in combination with other prevention supports including consistent condom use, has been associated with a relative reduction of $96 \%$ in the number of linked HIV-1 sexual transmissions in certain populations as compared with delayed therapy (4, $5)$.

In contrast, individuals who are unaware they have HIV infection, are more likely to have increased morbidity and mortality and unknowingly spread the virus.

In spite of the benefits associated with HIV testing, fear of testing positive, and fear of the anticipated discrimination that may come with a diagnosis of HIV can be barriers to testing (6). Reducing the number of undiagnosed HIV infections requires a balance between targeting tests to those most at risk with a less targeted approach directed to populations at "moderate risk" as well. Evidence demonstrates that many individuals who are, or perceived to be, outside of traditional high-risk populations are not being offered HIV testing $(7,8)$. As such they are sometimes diagnosed late in the progression of HIV disease, in spite of many prior interactions with the health system (8).

This is a summary of the HIV Screening and Testing Guide (9).

\section{Approach}

To inform the development of this guide, the Agency commissioned a literature review and consultations with provinces and territories, and key stakeholders, including people living with HIVIAIDS and other affected populations, academics, nurses, physicians, professional associations, non-governmental organizations, policy-makers, community workers, and legal and ethical experts. As a result, the recommendations outlined in the guide are based on the most up-to-date evidence and expert opinion.

Note, that this guide does not supersede any provincial/territorial legislative, regulatory, policy and practice requirements or professional guidelines that govern and inform the practice of care providers in their respective jurisdictions. As always, care providers should comply with practice requirements and local public health regulations when conducting HIV testing. This guide is designed to provide supplemental guidance in an effort to reduce the number of undiagnosed HIV infections in Canada. 


\section{Recommendations}

This guide is based on a number of guiding principles. The first is the importance of public health promotion and protection, which includes the need to integrate HIV testing with testing services for other related infections, such as sexually transmitted and blood borne infections and tuberculosis. The second is the importance of human rights, which includes the "3Cs" of HIV testing: counselling, informed consent, and confidentiality. The third is the principle of adaptability which reflects the need to apply recommendations with flexibility depending on the needs of both the client and the care provider.

\section{NORMALIZE HIV TESTING}

It is recommended that the consideration and discussion of HIV testing be made a component of routine periodic medical care. This recommendation is based upon two observations. First, evidence suggests that selfperceived risk does not always accurately reflect actual risk and, when combined with fear, may reduce the likelihood of patient-initiated requests for an HIV test (6). Second, there is good quality evidence demonstrating the benefits associated with normalising HIV testing as a means of overcoming multiple barriers associated with HIV testing (10) (Table 1). The testing process offers all clients with an opportunity to relieve any anxiety about an unknown HIV status and to establish a baseline as part of an individual's overall health care. Individuals involved in high risk behaviours should be offered HIV testing at least annually.

An in-depth comprehensive HIV behavioural risk assessment is not a pre-requisite for offering an HIV test. An assessment that the client understands how HIV is transmitted, the implications of testing (advantages and disadvantages), and how to interpret the test results is sufficient. A provider-initiated offer for HIV testing can begin with a brief explanation to the client on how HIV is transmitted: through unprotected sex, the sharing of drug-use equipment, and from a pregnant mother to her child. Clients can then consider their own situation and indicate whether they would like to have an HIV test.

It is helpful to have print and on-line resources to support client information needs to help inform discussions and decisions around HIV testing. For occasions when clients may not be able to accurately estimate their risk, a review of the clinical indications for HIV testing and risk factors for HIV may be helpful. (Table 2) In rare cases, an in depth behavioural risk assessment may be indicated (9). Advise the client that they have the right to decline the test. HIV testing remains voluntary and based on informed consent. Written consent is not necessary.

To overcome concerns relating to stigmatization, care providers should respect the cultural, sexual and gender diversities of clients by avoiding judgmental language, behaviours and attitudes and use appropriate information resources to reflect these diversities.

Individuals with a risk of HIV exposure, in an ongoing sexual relationship with a regular partner, can benefit from being offered HIV testing as a couple. Evidence indicates that couples who test together and are mutually aware of each other's results are more likely to adopt behaviours that will protect their partners, when compared to those who test alone (11). Couples' testing reduces HIV transmission among serodiscordant couples whose status is unknown to them, and reduces the risk of HIV transmission/acquisition with sexual partners external to the couple.

\section{PRE-TEST COUNSELLING}

It is useful to provide clients with HIV information in the waiting room to help prepare for pre-test discussions. Because clients who test positive are less likely to retain key information of post-test counselling, some post-test information is included as part of the pre-test discussion.

Communicate positive messaging around the benefits associated with the comfort of knowing one's negative HIV status or the benefits of an early diagnosis including available treatments and improved disease prognosis. Explain the window period and, if at risk behaviours have occurred in the previous three months, that follow-up testing may be required. Discuss steps the client can take to avoid acquisition or transmission of HIV and other STBBIs and that such steps should be undertaken until the completion of all testing (a client who continuously engages in risk 
behaviours remains in a continuous window period). Assure the testing client that his or her privacy or anonymity will be maintained and how. Explain potential limits to confidentiality including that a positive test result will be shared confidentially with the local public health department. Advise the client of the public health benefits of disclosing their HIV status to current and future partners in the event of a positive test result. Identify the client's post-test support needs. Test results should generally be provided in person, but it may be useful to have an agreed upon alternative to deliver HIV negative results and follow up recommendations, such as via a secure telephone call, letter or email.

\section{TESTING}

Algorithms for HIV testing have been developed to optimize the positive and negative predictive values; a full discussion of the tests goes beyond this guide. For information specific to your area, contact your local public health laboratory. It is important to note however, pre- and post-test counselling needs to be adapted to the generation and type of test. For example, more recent generations of tests have a reduced window period compared to earlier generations. Rapid testing, when compared to lab-based testing, involves an assessment of whether the client is prepared to receive a test result in the same session, and understands the meaning of a non-reactive test, a reactive test and the possibility of a false positive. Health Canada requires that rapid test kits are only to be used in settings where pre- and post-test HIV counselling is available.

\section{POST-TEST COUNSELLING}

Key messages for clients need to be tailored to the result of the HIV test and the individual circumstances. At times, pre- and post-test activities can be combined into a single session. The level of support required in any given testing situation may include information, discussion, counselling and/or referral. All clients should be offered referral service and supports, regardless of their test results, to support risk reduction measures. These services can be found by contacting local public health departments, provincial/territorial health information lines, AIDS hotlines or local crises centres.

\section{INDETERMINATE RESULT}

When the result is indeterminate, additional testing needs to be completed. Counsel the client to maintain risk reduction practices until all testing is complete. Review pre-test counselling messages.

\section{NEGATIVE RESULT}

For the majority of HIV tests performed, results will be negative. Clients should be made aware of the window period, and if any risk behaviours occurred in the three months prior to the test, follow-up testing is recommended. When negative test results are confirmed, this is an opportunity to remind clients of those practices that can help them maintain an HIV-negative status. Clients testing negative who are part of a couple should be encouraged to discuss HIV testing with their sexual/drug use partners.

\section{POSITIVE RESULT}

An HIV positive test result should always be provided in person and ideally by the initial care provider. Care providers should be prepared in advance by having information resources and support referrals at the ready for the client and be able to spend sufficient time to discuss the results and answer any immediate questions the client might have. Frequently, clients will only hear the positive test result, so it can be helpful to schedule a follow-up appointment within two weeks so further discussions can occur.

Clients should be reassured that their privacy and confidentiality will be protected. Note that with proper care and treatment, people with HIV can live long, healthy and active lives and clients should be made aware that HIV is now considered a chronic manageable condition. Advise the client about strategies for managing HIV and link them to care. If not already completed with the HIV test, the client should be tested for other STIs, hepatitis B and C, and tuberculosis. Baseline testing, such as CD4, plasma viral load, and drug resistance should be ordered. It is imperative that clients be informed about how to prevent the further spread of the virus. 
A partner notification plan should be developed with the client so previous and current partners can be notified of their need to be tested. There are several options for notification. With the consent of the client, Public Health services can assist in anonymous notification of potentially exposed sexual and drug-equipment sharing partners who have been identified by the client. Alternatively, the testing provider can assist with anonymous notification or the client may wish to contact his or her partners directly. Some clients may prefer a combination of the above, depending on the contact. In cases where sexual partners have met over the internet or only exchanged an email address or username, web-based tools, such as inSPOT may help increase partner notification. There should be an agreed upon time period for confirming that partners have been notified.

Provide risk reduction information to prevent transmission of the virus as a critical element in post-test procedures. In circumstances where the care provider is not able to offer in-depth risk-reduction information, a referral to riskreduction services should be provided.

Offer client referrals to specialized counselling services that are equipped to provide newly diagnosed individuals with the specific supports and resources that they need to manage their health and wellness. HIVIAIDS hotlines are available in each province and territory in Canada.

\section{Conclusion}

This guide was designed to support care providers to offer HIV testing routinely and flexibly to detect previously undiagnosed cases of HIV. This can decrease HIV transmission and improve productivity, disease prognosis, and quality of life for people living with HIV. Once the diagnosis is made, supports and ongoing care is critical.

\section{References}

1. Public Health Agency of Canada. Summary: Estimates of HIV prevalence and incidence in Canada, 2011. Ottawa, ON: .

2. Jain V, Deeks SG. When to start antiretroviral therapy. Current HIVIAIDS Reports. 2010;7(2):60-8.

3. Marks G, Crepaz N, Senterfitt JW, Janssen RS. Meta-analysis of high-risk sexual behavior in persons aware and unaware they are infected with HIV in the United States: Implications for HIV prevention programs. J Acquir Immune Defic Syndr. 2005 Aug 1;39(4):446-53.

4. Attia S, Egger M, Müller M, Zwahlen M, Low N. Sexual transmission of HIV according to viral load and antiretroviral therapy: Systematic review and meta-analysis. AIDS. 2009;23(11):1397-404.

5. Cohen MS, Chen YQ, McCauley M, Gamble T, Hosseinipour MC, Kumarasamy N, et al. Prevention of HIV-1 infection with early antiretroviral therapy. N Engl J Med. 2011;365(6):493-505.

6. Deblonde J, De Koker P, Hamers FF, Fontaine J, Luchters S, Temmerman M. Barriers to HIV testing in Europe: A systematic review. Eur J Public Health. 2010;20(4):422-32.

7. Burke RC, Sepkowitz KA, Bernstein KT, Karpati AM, Myers JE, Tsoi BW, et al. Why don't physicians test for HIV? A review of the US literature. AIDS. 2007;21(12):1617-24.

8. Girardi E, Sabin CA, Monforte AD. Late diagnosis of HIV infection: Epidemiological features, consequences and strategies to encourage earlier testing. J Acquir Immune Defic Syndr. 2007;46(SUPPL. 1):S3-8.

9. Public Health Agency of Canada. Human immunodeficiency virus HIV screening and testing guide. Canada: 2013. http://publications.gc.ca/collections/collection_2013/aspc-phac/HP40-76-2012-eng.pdf

10. European Centre for Disease Prevention and Control. HIV testing: Increasing uptake and effectiveness in the European Union. Stockholm: ECDC; 2010.

11. World Health Organization. Guidance on couples HIV testing and counselling including antiretroviral therapy for treatment and prevention in serodiscordant couples: Recommendations for a public health approach. Geneva: WHO; 2012. 


\section{Acknowledgements}

The Public Health Agency of Canada (PHAC) would like to thank the members of the Communicable and Infectious Disease Steering Committee, Federal/Provincial/Territorial Advisory Committee on AIDS, the Canadian Guidelines on Sexually Transmitted Infections Expert Working Group, the Council of Chief Medical Officers of Health, the Canadian Public Health Laboratory Network and the Canadian Association of HIV Clinical Laboratory Specialists for their input into this Guide. PHAC would also like to acknowledge the many staff of the Centre for Communicable Diseases and Infection Control and the National Microbiology Laboratory who contributed to the development of this document.

\section{Conflict of interest statement}

There are no conflicts of interest to declare.

\section{Funding}

This work was supported by the Public Health Agency of Canada.

\section{TABLE 1. BARRIERS TO HIV TESTING AND RECOMMENDATIONS IN THE GUIDE TO ADDRESS THEM}

Inability to accurately assess levels of risk for exposure to HIV by some clients and providers

Lack of comfort discussing HIV testing and knowledge about HIV among some clients and providers

Provider time constraints for risk assessments and preand post-test counselling

Cumbersome consent procedures

Fear of stigma and discrimination associated with risk behaviours and/or testing HIV-positive
Normalise HIV testing; simplify risk assessments; make the consideration of an HIV test part of periodic routine medical care.

Normalise HIV testing; simplify risk assessments; make the consideration of an HIV test a part of periodic routine medical care.

Simplify risk assessments; streamline the provision of pre-test information using print, video, mobile and web-based resources; alternate approaches offered to provide negative results.

Verbal consent for HIV testing, as with other tests, is sufficient; testing remains voluntary.

Normalise HIV testing and simplify risk assessment to reduce discomfort and stigma and increase uptake of testing; emphasize HIV as a chronic manageable condition and the benefits of treatment to reduce fear of HIV diagnosis.

\section{TABLE 2. CLINICAL INDICATIONS FOR HIV TESTING AND RISK FACTORS FOR HIV INFECTION}

\section{CLINICAL INDICATIONS FOR HIV TESTING}

1. Individuals requesting an HIV test.

2. Individuals with symptoms and signs of HIV infection.

3. Individuals with illnesses associated with a weakened immune system or a diagnosis of tuberculosis.

4. Unprotected anal or vaginal intercourse or use of shared drug equipment with a partner whose HIV status is known to be positive.

5. Pregnant or planning a pregnancy; and their partners as appropriate.

6. Victims of sexual assault. 
FACTORS THAT INCREASE RISK FOR HIV INFECTION

1. Sexually active but no history of being tested for HIV.

2. Use of shared drug equipment with a partner whose HIV status is unknown.

3. Unprotected anal or vaginal intercourse with a partner whose HIV status is unknown.

4. Multiple and/or anonymous sexual partnering.

5. For men, a history of sex with other men.

6. Diagnosis of other STI, hepatitis B or C.

7. Sexual activity, sharing of drug-use equipment, or receipt of blood or blood products for people originating from, or who have travelled to, regions where HIV is endemic.

8. Receipt of blood or blood products in Canada prior to November 1985. 